\title{
AN APPLICATION OF INTERPOLATION THEORY TO RENORMING OF LORENTZ-KARAMATA TYPE SPACES
}

\author{
Pedro Fernández-Martínez and Teresa M. Signes \\ Universidad de Murcia, Facultad de Informática, Departamento de Matemática Aplicada \\ Campus de Espinardo, 30071 Espinardo (Murcia), Spain; pedrofdz@um.es \\ Universidad de Murcia, Facultad de Informática, Departamento de Matemática Aplicada \\ Campus de Espinardo, 30071 Espinardo (Murcia), Spain; tmsignes@um.es
}

\begin{abstract}
We apply techniques of interpolation of operators acting on cones of measurable functions to prove new characterizations of Lorentz-Karamata type spaces.
\end{abstract}

\section{Introduction}

Lorentz-Karamata spaces is a class of function spaces that comprises Lebesgue spaces, Lorentz spaces, Lorentz-Zygmund spaces and even generalized Lorentz-Zygmund spaces. This class offers not only a more general and unified insight for these families of spaces but also provides a framework in which it is easier to appreciate the central issues of different results. We refer to [10] and [16] for more information on this topic.

There is a standard way of defining Lorentz-Karamata spaces. However, some papers that show alternative characterizations of Lorentz and Lorentz-Karamata spaces by means of equivalent quasi-norms have recently appeared. See [18], [17] and [11] for Lorentz spaces and [13] for Lorentz-Karamata spaces. In the present paper we extend those results to a wider class of spaces for which we find equivalent quasi-norms that characterize them.

The techniques we use to establish these equivalences are rather different to those that appear in [18], [17], [11] or [13]. We combine interpolation of operators acting on cones of function spaces with some of the ideas sketched in [13]. Thus, we achieve more general theorems through simpler proofs that make easier to see the most relevant aspects of these problems.

In order to be more precise about our results let $(\Omega, \mu)$ be a $\sigma$-finite measure space with a non-atomic measure. Given a slowly varying function $b$, see Definition 2.2 below, a real parameter $1 \leq p \leq \infty$ and a rearrangement invariant space $E$, the Lorentz-Karamata type space $L_{p, E, b}$ consists of all measurable functions on $\Omega$ for which the (quasi-)norm

$$
\|f\|_{p, E, b}=\left\|t^{1 / p} b(t) f^{*}(t)\right\|_{\widetilde{E}}
$$

is finite. Here $f^{*}$ stands for the decreasing rearrangement of the function $f$.

doi:10.5186/aasfm.2014.3911

2010 Mathematics Subject Classification: Primary 46B70, 46E30, 47B38.

Key words: Function spaces, function cones, ultrasymmetric spaces, interpolation theory, Lorentz-Karamata spaces

Partially supported by Ministerio de Ciencia e Innovación (MTM2010-15814) and by Región de Murcia, Fundación Séneca 08791/PI/08. 
We prove that, given $\alpha, \beta \in \mathbf{R}$ with $\beta>0, a$ and $b$ slowly varying functions and $E, F$ rearrangement invariant spaces, the following equivalence

$$
\left\|t^{\beta} b(t)\right\| u^{\alpha} a(u) f^{*}(u)\left\|_{\widetilde{F}(t, \infty)}\right\|_{\widetilde{E}} \sim\left\|t^{\alpha+\beta} a(t) b(t) f^{*}(t)\right\|_{\widetilde{E}}
$$

holds for any measurable function $f$ on $(\Omega, \mu)$. For the case $\beta<0$ we establish the equivalence

$$
\left\|t^{\beta} b(t)\right\| u^{\alpha} a(u) f^{*}(u)\left\|_{\widetilde{F}(0, t)}\right\|_{\widetilde{E}} \sim\left\|t^{\alpha+\beta} a(t) b(t) f^{*}(t)\right\|_{\widetilde{E}}
$$

for any measurable function $f$. Here the spaces $\widetilde{E}$ and $\widetilde{F}$ are rearrangement invariant spaces with respect to the homogeneous measure on $(0, \infty), d t / t$. See $\S 2.1$ below for a detailed description.

These equivalences are useful in different contexts, for example in the study of the boundedness of Hardy-type operators $T f(t)=v(t) \int_{0}^{t} u(s) f(s) d s$. To illustrate this we consider the operator

$$
T f(t)=\frac{v(t)}{t^{1 / q+\alpha}} \int_{0}^{t} u(s) f(s) d s
$$

where $v$ is a slowly varying function and $0<\alpha$. Then for $1 \leq p \leq q \leq \infty$, the operator

$$
T: L_{p}(0, \infty) \longrightarrow L_{q}(0, \infty)
$$

is bounded if and only if

$$
\left\|t^{-\alpha} v(t)\right\| s^{1 / p^{\prime}} u(s)\left\|_{\widetilde{L}_{p^{\prime}}(0, t)}\right\|_{\widetilde{L}_{\infty}(0, \infty)}<\infty .
$$

See [10], Thm. 2.2.1 combined with Lemma 2.3 of [15]. Using the above equivalences we have that for $1 \leq p \leq q \leq \infty$ the operator

$$
T: L_{p}(0, \infty) \longrightarrow L_{q}(0, \infty)
$$

is bounded if and only if

$$
t^{1 / p^{\prime}-\alpha} v(t) u(t) \in L_{\infty} .
$$

The paper is organized as follows. Section 2 contains the basic notions and definitions concerning rearrangement invariant spaces, interpolation on function cones, Marcinkiewicz couples and Lorentz-Karamata type spaces. In Section 3 we can find the main results of the paper, Theorems 3.6 and 3.7, along with some interesting properties of slowly varying functions. Section 4 extends the results shown in [13] for maximal fractional operators and operators of Riesz potential type to the context of Lorentz-Karamata type spaces.

Through this paper we will use the notation $a \lesssim b$ to indicate that $a \leq C b$ for some constant $C>0$. Similarly we will write $a \gtrsim b$ if there exists a constant $C>0$ such that $a \geq C b$. When $a \lesssim b$ and $b \lesssim a$ we will write $a \sim b$.

\section{Preliminaries}

In this section we recall the basic notions and definitions of rearrangement invariant function spaces, real interpolation of operators acting on function cones, slowly varying functions and Lorentz-Karamata spaces. 
2.1. Rearrangement invariant spaces. A Banach function space $E$ over the measure space $(\Omega, \mu)$ with the Fatou property is called rearrangement invariant (r.i.) if, for any two measurable functions $f$ and $g$, the conditions

$$
g \in E \quad \text { and } \quad f^{*}(t) \leq g^{*}(t) \text { a.e. }
$$

imply $f \in E$ and $\|f\|_{E} \leq\|g\|_{E}$. The functions $f^{*}$ and $g^{*}$ are the non-decreasing rearrangement of $f$ and $g$ respectively. See [1] for a more detailed description of r.i. spaces.

Every r.i. space over a resonant measure space $(\Omega, \mu)$ can be identified with an r.i. space over $(0, \infty)$ with the Lebesgue measure, its representation space. See [1] Thm. 4.10. Thus in what follows $E$ (and $E_{0}, E_{1}$ ) will stand for an r.i. space over the measure space $(0, \infty)$ with the Lebesgue measure, unless otherwise stated. We will also work with the homogeneous measure $\frac{d t}{t}$ on $(0, \infty)$ and we will denote by $\widetilde{L}_{1}$ and $\widetilde{L}_{\infty}$ the Lebesgue spaces defined by the norms

$$
\|f\|_{\widetilde{L}_{1}}=\int_{0}^{\infty}|f(t)| \frac{d t}{t} \quad \text { and } \quad\|f\|_{\widetilde{L}_{\infty}}=\|f\|_{L_{\infty}} .
$$

Given any r.i. space $E$, we define its counterpart $\widetilde{E}$ aided by the spaces $\widetilde{L}_{1}$ and $\widetilde{L}_{\infty}$ in the following way. As an r.i. space $E$ can be obtained through an exact interpolation method from the couple $\left(L_{1}, L_{\infty}\right)$. More precisely, there exists an exact real interpolation method, $\mathcal{F}$, such that $E=\mathcal{F}\left(L_{1}, L_{\infty}\right)$. See [1] for more information. We denote by $\underset{\widetilde{E}}{\widetilde{E}}$ the space obtained by applying the same interpolation method $\mathcal{F}$ to the couple $\left(\widetilde{L}_{1}, \widetilde{L}_{\infty}\right)$. Namely

$$
\widetilde{E}=\mathcal{F}\left(\widetilde{L}_{1}, \widetilde{L}_{\infty}\right)
$$

It may be worthy to mention that the spaces $E$ and $\widetilde{E}$ are connected by the following relations:

$$
\|f\|_{\widetilde{E}(0,1)}=\left\|f\left(e^{-u}\right)\right\|_{E(0, \infty)} \quad \text { and } \quad\|f\|_{\widetilde{E}(1, \infty)}=\left\|f\left(e^{u}\right)\right\|_{E(0, \infty)} .
$$

In particular $f \in \widetilde{E}$ if and only if $f\left(e^{-u}\right)$ and $f\left(e^{u}\right), u>0$, belong to $E$. In that case we have the following equivalence

$$
\|f\|_{\widetilde{E}} \sim\left\|f\left(e^{-u}\right)\right\|_{E}+\left\|f\left(e^{u}\right)\right\|_{E} .
$$

2.2. Interpolation on function cones. Interpolation of operators acting on cones of measurable functions has been a subject of interest in the past years. The first author using these techniques was Sagher in his paper [20], where Fourier series with positive coefficients were studied. Later, some other papers on this topic were published. See, for instance, [8], [6] or [4].

A function cone $Q$ is a subset of non-negative measurable functions on $\Omega$ such that

$$
Q+Q \subset Q, \text { and } \lambda Q \subset Q \text {, for all } \lambda \geq 0 \text {. }
$$

One of the most common examples is the cone of the decreasing (i.e. non-negative and non-increasing) functions on $\mathbf{R}^{+}$.

If $\bar{E}=\left(E_{0}, E_{1}\right)$ is a couple of Banach function spaces, and $Q$ a function cone on $(\Omega, \mu)$, we may consider two different $K$-functionals relative to $\bar{E}$. The classical 
$K$-functional on $\bar{E}$

$$
K(t, f ; \bar{E})=\inf \left\{\left\|f_{0}\right\|_{0}+t\left\|f_{1}\right\|_{1} ; f=f_{0}+f_{1}, f_{i} \in E_{i}, i=0,1\right\},
$$

and the $K^{Q}$-functional on $\bar{E} \cap Q=\left(E_{0} \cap Q, E_{1} \cap Q\right)$ defined as

$$
K^{Q}(t, f ; \bar{E} \cap Q)=\inf \left\{\left\|f_{0}\right\|_{0}+t\left\|f_{1}\right\|_{1} ; f=f_{0}+f_{1}, f_{i} \in E_{i} \cap Q, i=0,1\right\} .
$$

These $K$-functionals generate both interpolation spaces for the couples $\bar{E}$ and $\bar{E} \cap Q$. More precisely, if $\Phi$ is an admissible Banach lattice $(\min \{1, t\}, t>0$ belongs to $\Phi)$ we define the space

$$
\left(E_{0}, E_{1}\right)_{\Phi}^{K}=\left\{f \in E_{0}+E_{1} \text { s.t. } K(\cdot, f, E) \in \Phi\right\}
$$

with norm

$$
\|f\|_{K_{\Phi}(\bar{E})}=\|K(\cdot, f, \bar{E})\|_{\Phi}
$$

and the space

$$
\left(E_{0} \cap Q, E_{1} \cap Q\right)_{\Phi}^{K^{Q}}=\left\{f \in\left(E_{0} \cap Q\right)+\left(E_{1} \cap Q\right) \text { s.t. } K^{Q}(\cdot, f, E) \in \Phi\right\}
$$

with norm

$$
\|f\|_{K_{\Phi}^{Q}(\bar{E})}=\left\|K^{Q}(\cdot, f, \bar{E})\right\|_{\Phi} .
$$

We refer to [3] for a complete description of $K$-spaces.

Clearly, for any $f \in\left(E_{0} \cap Q\right)+\left(E_{1} \cap Q\right)$,

$$
K(t, f ; \bar{E}) \leq K^{Q}(t, f ; \bar{E} \cap Q), \quad t>0,
$$

and thus

$$
\left(E_{0} \cap \bar{E}, E_{1} \cap \bar{E}\right)_{\Phi}^{K^{Q}} \subset\left(E_{0}, E_{1}\right)_{\Phi}^{K} \cap Q .
$$

Examples showing that the equality

$$
\left(E_{0} \cap Q, E_{1} \cap Q\right)_{\Phi}^{K^{Q}}=\left(E_{0}, E_{1}\right)_{\Phi}^{K} \cap Q
$$

does not always hold are easy to obtain. We may consider the cone $Q$ of measurable decreasing functions on $(0, \infty)$ and the couple of weighted $L_{1}$-spaces, $\left(L_{1}, L_{1}\left(e^{-x}\right)\right)$. Then interpolation by the real method yields

$$
\left(L_{1}, L_{1}\left(e^{-x}\right)\right)_{\theta, 1} \cap Q \neq\left(L_{1} \cap Q, L_{1}\left(e^{-x}\right) \cap Q\right)_{\theta, 1} .
$$

See [6]. This motivates the following definition.

Definition 2.1. We say that $\bar{E}=\left(E_{0}, E_{1}\right)$ is a Marcinkiewicz couple for the function cone $Q$ if the equivalence

$$
\|f\|_{K_{\Phi}(\bar{E})} \sim\|f\|_{K_{\Phi}^{Q}(\bar{E})}
$$

holds for all admissible Banach lattices $\Phi$ on $\left(\mathbf{R}^{+}, d t / t\right)$ and the equivalence constant does not depend on $f \in\left(E_{0} \cap Q\right)+\left(E_{1} \cap Q\right)$. In this case

$$
\left(E_{0} \cap Q, E_{1} \cap Q\right)_{\Phi}^{K^{Q}}=\left(E_{0}, E_{1}\right)_{\Phi}^{K} \cap Q .
$$


A sufficient condition for $\bar{E}$ to be a Marcikiewicz couple for the cone $Q$ is that

$$
K^{Q}\left(t, f ; E_{0} \cap Q, E_{1} \cap Q\right) \lesssim K\left(t, f ; E_{0}, E_{1}\right)
$$

with inequality constant only depending on $\bar{E}$.

Henceforth $Q$ will be the cone of decreasing functions on $\mathbf{R}^{+}$. The couple $\left(L_{1}(\mu), L_{\infty}(\mu)\right)$ is a Marcinkiewicz couple for $Q$, see [5], [6] and [7]. Therefore

$$
\left(L_{1}(\mu) \cap Q, L_{\infty}(\mu) \cap Q\right)_{\Phi}^{K^{Q}}=\left(L_{1}(\mu), L_{\infty}(\mu)\right)_{\Phi}^{K} \cap Q
$$

for any admissible Banach lattice $\Phi$.

2.3. Generalized Lorentz-Karamata spaces. Lorentz-Karamata spaces were introduced by Edmunds, Pick and Kerman in [14]. This class includes Lebesgue, Lorentz, Lorentz-Zygmund and generalised Lorentz-Zygmund spaces. A detailed description of these spaces, as well as examples of their usefulness, can be found in [16] and [10].

Definition 2.2. We say that a positive Lebesgue measurable function $b, 0 \not \equiv$ $b \not \equiv \infty$, is slowly varying on $(0, \infty)$ if, for each $\varepsilon>0$, the function $t^{\varepsilon} b(t), t>0$, is almost increasing on $(0, \infty)$ while the function $t^{-\varepsilon} b(t), t>0$, is almost decreasing on $(0, \infty)$.

We refer to [2] for examples and more information on slowly varying functions. Next we define Lorentz-Karamata type spaces.

Definition 2.3. Let $0<p \leq \infty$ be a real parameter, $b$ a slowly varying function and $E$ a r.i. space. The Lorentz-Karamata type space $L_{p, E, b}$ is the set of all measurable functions $f$ on $(\Omega, \mu)$ such that

$$
\|f\|_{p, E, b}:=\left\|t^{1 / p} b(t) f^{*}(t)\right\|_{\widetilde{E}}<\infty .
$$

In case we choose $b \equiv 1$ and $E=L_{q}$, the Lorentz-Karamata space $L_{p, L_{q}, b}$ coincides with the classical Lorentz space $L_{p, q}$. If, on the contrary, we choose $b(t)=$ $\ell^{\alpha}(t)=(1+|\log t|)^{\alpha}, t>0$, and keep $E=L_{q}$, the Lorentz-Karamata space $L_{p, L_{q}, \ell^{\alpha}(t)}$ coincides with the Lorentz-Zygmund space $L^{p, q}(\log L)^{\alpha}$. In general, if we restrict $E$ to the class of the classical Lebesgue spaces $L_{q}$ we obtain the collection of LorentzKaramata spaces as defined in [13] or [16].

\section{Renorming of Lorentz-Karamata type spaces}

In this section we present the main theorems of the paper. Let us begin with some auxiliary results that show different features of slowly varying functions. The following lemma can be found in [15].

Lemma 3.1. Let a be a slowly varying function, $E$ an r.i. space on $(0, \infty)$ and $\alpha>0$. Then for $t>0$

$$
\left\|s^{\alpha} a(s)\right\|_{\widetilde{E}(0, t)} \sim t^{\alpha} a(t) \quad \text { and } \quad\left\|s^{-\alpha} a(s)\right\|_{\widetilde{E}(t, \infty)} \sim t^{-\alpha} a(t) .
$$

In the same fashion of Lemma 3.1, next results provide useful estimates for the norm of some families of functions described through slowly varying functions.

Lemma 3.2. Let a be a slowly varying function, and let $\alpha \in \mathbf{R}$. Then for $t>0$

$$
\left\|s^{\alpha} a(s)\right\|_{\widetilde{E}(t, 2 t)} \sim t^{\alpha} a(t) .
$$


Proof. We prove the equivalence for $\alpha \geq 0$. Using Lemma 3.1

$$
\left\|s^{\alpha} a(s)\right\|_{\widetilde{E}(t, 2 t)} \leq\left\|\frac{s^{\alpha+1}}{t} a(s)\right\|_{\widetilde{E}(t, 2 t)} \leq \frac{1}{t}\left\|s^{\alpha+1} a(s)\right\|_{\widetilde{E}(0,2 t)} \sim t^{\alpha} a(t) .
$$

For the reverse inequality we have

$$
\begin{aligned}
\left\|s^{\alpha} a(s)\right\|_{\widetilde{E}(t, 2 t)} & \geq\left\|\frac{s^{\alpha+1}}{2 t} a(s)\right\|_{\widetilde{E}(t, 2 t)} \gtrsim \frac{t^{\alpha+1}}{t} a(t)\left\|\chi_{(t, 2 t)}\right\|_{\widetilde{E}} \\
& \sim t^{\alpha} a(t) \varphi_{E}(\ln 2) \sim t^{\alpha} a(t) .
\end{aligned}
$$

The case $\alpha<0$ is proved by using similar arguments.

Lemma 3.3. Let $f \in Q, \alpha \in \mathbf{R}$ and let a be a slowly varying function. Then for any r.i. space $E$ and $t>0$

$$
\left\|u^{\alpha} a(u) f(u)\right\|_{\widetilde{E}(0, t)} \lesssim \int_{0}^{t} u^{\alpha} a(u) f(u) \frac{d u}{u} .
$$

Proof. Consider the following restrictions of the identity operator

$$
\begin{aligned}
& \widetilde{L}_{1}\left(u^{\alpha} a(u),(0, t)\right) \cap Q \stackrel{\mathrm{Id}}{\longrightarrow} \widetilde{L}_{1}\left(u^{\alpha} a(u),(0, t)\right), \\
& \widetilde{L}_{1}\left(u^{\alpha} a(u),(0, t)\right) \cap Q \stackrel{\mathrm{Id}}{\longrightarrow} \widetilde{L}_{\infty}\left(u^{\alpha} a(u),(0, t)\right),
\end{aligned}
$$

where $\widetilde{L}_{1}\left(u^{\alpha} a(u),(0, t)\right)$ and $\widetilde{L}_{\infty}\left(u^{\alpha} a(u),(0, t)\right)$ denote the weighted Lebesgue spaces defined by the norms

$$
\|f\|_{\widetilde{L}_{1}\left(u^{\alpha} a(u),(0, t)\right)}=\int_{0}^{t} u^{\alpha} a(u)|f(u)| \frac{d u}{u}
$$

and

$$
\|f\|_{\widetilde{L}_{\infty}\left(u^{\alpha} a(u),(0, t)\right)}=\sup _{t \in(0, t)} u^{\alpha} a(u)|f(u)| .
$$

Restriction (2) is obviously bounded. In order to check boundedness of restriction (3) we use Lemma 3.1 and the fact that $f$ is a decreasing function. In case $\alpha>0$

$$
\begin{aligned}
\left\|u^{\alpha} a(u) f(u)\right\|_{\widetilde{L}_{\infty}(0, t)} & =\sup _{0<u<t} u^{\alpha} a(u) f(u) \sim \sup _{0<u<t} \int_{0}^{u} s^{\alpha} a(s) \frac{d s}{s} f(u) \\
& \leq \sup _{0<u<t} \int_{0}^{u} s^{\alpha} a(s) f(s) \frac{d s}{s}=\|f\|_{\widetilde{L}_{1}\left(u^{\alpha} a(u),(0, t)\right)} .
\end{aligned}
$$

For $\alpha \leq 0$

$$
\begin{aligned}
\left\|u^{\alpha} a(u) f(u)\right\|_{\widetilde{L}_{\infty}(0, t)} & \sim \sup _{0<u<t} \int_{0}^{u} s a(s) \frac{d s}{s} \frac{f(u)}{u^{1-\alpha}} \\
& \lesssim \sup _{0<u<t} \int_{0}^{u} s^{\alpha} a(s) f(s) \frac{d s}{s}=\|f\|_{\widetilde{L}_{1}\left(u^{\alpha} a(u),(0, t)\right)} .
\end{aligned}
$$

It is important to mention that the equivalence and inequalities constants in the previous equation do not depend on $t>0$. Then we can interpolate the identity operator in (2) and (3) to obtain that the restrictions

$$
\widetilde{L}_{1}\left(u^{\alpha} a(u),(0, t)\right) \cap Q \stackrel{\mathrm{Id}}{\longrightarrow} \widetilde{E}\left(u^{\alpha} a(u),(0, t)\right)
$$


are uniformly bounded for $t>0$. Therefore

$$
\left\|u^{\alpha} a(u) f(u)\right\|_{\widetilde{E}(0, t)} \lesssim \int_{0}^{t} u^{\alpha} a(u) f(u) \frac{d u}{u}, \quad t>0 .
$$

Lemma 3.4. Let $a$ be any slowly varying function, $E$ an r.i. space and let $\alpha \in \mathbf{R}$. Then for any $f \in Q$ and $t>0$

$$
\left\|s^{\alpha} a(s) f(s)\right\|_{\widetilde{E}(t, \infty)} \lesssim \int_{\frac{t}{2}}^{\infty} s^{\alpha} a(s) f(s) \frac{d s}{s} .
$$

The inequality constant does not depend on $f \in Q$ nor $t>0$.

Proof. Consider the following restrictions of the identity operator

$$
\begin{aligned}
& \widetilde{L}_{1}\left(s^{\alpha} a(s),\left(\frac{t}{2}, \infty\right)\right) \cap Q \stackrel{\mathrm{Id}}{\longrightarrow} \widetilde{L}_{1}\left(s^{\alpha} a(s),(t, \infty)\right), \\
& \widetilde{L}_{1}\left(s^{\alpha} a(s),\left(\frac{t}{2}, \infty\right)\right) \cap Q \stackrel{\mathrm{Id}}{\longrightarrow} \widetilde{L}_{\infty}\left(s^{\alpha} a(s),(t, \infty)\right) .
\end{aligned}
$$

We focus on the boundedness of the second restriction. Lemma 3.2 and the monotonicity of $f$ yield

$$
\begin{aligned}
\|f\|_{\widetilde{L}_{\infty}\left(s^{\alpha} a(s),(t, \infty)\right)} & =\sup _{t<s<\infty} s^{\alpha} a(s) f(s) \sim \sup _{t<s<\infty} \int_{\frac{s}{2}}^{s} u^{\alpha} a(u) \frac{d u}{u} f(s) \\
& \leq \sup _{t<s<\infty} \int_{\frac{s}{2}}^{s} u^{\alpha} a(u) f(u) \frac{d u}{u} \leq \int_{\frac{t}{2}}^{\infty} u^{\alpha} a(u) f(u) \frac{d u}{u} \\
& =\|f\|_{\widetilde{L}_{1}\left(s^{\alpha} a(s),\left(\frac{t}{2}, \infty\right)\right)} .
\end{aligned}
$$

Now we interpolate in (4) and (5), using the real method that generates the space $E$ from the couple $\left(L_{1}, L_{\infty}\right)$, to establish that

$$
\widetilde{L}_{1}\left(s^{\alpha} a(s),\left(\frac{t}{2}, \infty\right)\right) \cap Q \stackrel{\mathrm{Id}}{\longrightarrow} \widetilde{E}\left(s^{\alpha} a(s),(t, \infty)\right)
$$

is a bounded operator.

Finally we collect the following lemma from [15] that deals with Hardy-type inequalities in the context of r.i. spaces.

Lemma 3.5. Let $E$ be an r.i. space on $(0, \infty), b$ a slowly varying function and $\alpha>0$. Then for each positive measurable function $f$ on $(0, \infty)$

$$
\left\|t^{-\alpha} b(t) \int_{0}^{t} f(s) d s\right\|_{\widetilde{E}} \lesssim\left\|t^{1-\alpha} b(t) f(t)\right\|_{\widetilde{E}}
$$

and

$$
\left\|t^{\alpha} b(t) \int_{t}^{\infty} f(s) d s\right\|_{\widetilde{E}} \lesssim\left\|t^{1+\alpha} b(t) f(t)\right\|_{\widetilde{E}}
$$

Now we are in a position to establish the main results of the paper. Their antecedents can be found in the papers [17] and [13]. Although the basics ideas behind next theorems are contained in those papers, the proofs we present here use different techniques and apply to a larger class of spaces.

Theorem 3.6. Let $a$ and $b$ be slowly varying functions, and let $E$ and $F$ be r.i. spaces. Choose $\alpha, \beta \in \mathbf{R}$ with $\beta>0$. Then the equivalence

$$
\left\|t^{\beta} b(t)\right\| u^{\alpha} a(u) f(u)\left\|_{\widetilde{F}(t, \infty)}\right\|_{\widetilde{E}} \sim\left\|t^{\alpha+\beta} a(t) b(t) f(t)\right\|_{\widetilde{E}}
$$


holds for any $f \in Q$.

Proof. Using Lemma 3.2 and the monotonicity of $f$ we find that

$$
\left\|u^{\alpha} a(u) f(u)\right\|_{\widetilde{F}(t, \infty)} \geq\left\|u^{\alpha} a(u) f(u)\right\|_{\widetilde{F}(t, 2 t)} \gtrsim f(2 t)(2 t)^{\alpha} a(2 t) .
$$

Hence

and therefore

$$
t^{\beta} b(t)\left\|u^{\alpha} a(u) f(u)\right\|_{\widetilde{F}(t, \infty)} \gtrsim(2 t)^{\alpha+\beta} a(2 t) b(2 t) f(2 t),
$$

$$
\left\|t^{\beta} b(t)\right\| u^{\alpha} a(u) f(u)\left\|_{\widetilde{F}(t, \infty)}\right\|_{\widetilde{E}} \gtrsim\left\|t^{\alpha+\beta} a(t) b(t) f(t)\right\|_{\widetilde{E}} .
$$

Note that we have used that $\|f(t)\|_{\widetilde{E}}=\|f(2 t)\|_{\widetilde{E}}$ for any $f \in \widetilde{E}$.

Let's prove the converse inequality. Using Lemma 3.4 and Hardy inequality (7) we can establish that

$$
\begin{aligned}
\left\|t^{\beta} b(t)\right\| u^{\alpha} a(u) f(u)\left\|_{\widetilde{F}(t, \infty)}\right\|_{\widetilde{E}} & \lesssim\left\|\left(\frac{t}{2}\right)^{\beta} b\left(\frac{t}{2}\right) \int_{\frac{t}{2}}^{\infty} s^{\alpha} a(s) f(s) \frac{d s}{s}\right\|_{\widetilde{E}} \\
& \lesssim\left\|\left(\frac{t}{2}\right)^{\beta} b\left(\frac{t}{2}\right)\left(\frac{t}{2}\right)^{\alpha} a\left(\frac{t}{2}\right) f\left(\frac{t}{2}\right)\right\|_{\widetilde{E}} \\
& \sim\left\|t^{\alpha+\beta} a(t) b(t) f(t)\right\|_{\widetilde{E}} .
\end{aligned}
$$

This completes the proof.

Next theorem allows the parameter $\beta$ to be negative. In such a case we work with the r.i. space $\widetilde{F}(0, t)$.

Theorem 3.7. Let $a$ and $b$ be slowly varying functions, and let $E$ and $F$ be r.i. spaces. Choose $\alpha, \beta \in \mathbf{R}$ with $\beta<0$. Then for any $f \in Q$, we have that

$$
\left\|t^{\beta} b(t)\right\| u^{\alpha} a(u) f(u)\left\|_{\widetilde{F}(0, t)}\right\|_{\widetilde{E}} \sim\left\|t^{\alpha+\beta} a(t) b(t) f(t)\right\|_{\widetilde{E}} .
$$

In particular

$$
\left\|b(t) f^{* *}(t)\right\|_{\widetilde{E}} \sim\left\|b(t) f^{*}(t)\right\|_{\widetilde{E}} .
$$

Proof. Hardy inequality (6) and Lemma 3.3 yield

$$
\begin{aligned}
& \left\|t^{\beta} b(t)\right\| u^{\alpha} a(u) f(u)\left\|_{\widetilde{F}(0, t)}\right\|_{\widetilde{E}} \lesssim\left\|t^{\beta} b(t) \int_{0}^{t} u^{\alpha} a(u) f(u) \frac{d u}{u}\right\|_{\widetilde{E}} \\
& \lesssim\left\|t^{\alpha+\beta} b(t) a(t) f(t)\right\|_{\widetilde{E}} .
\end{aligned}
$$

In order to prove the converse inequality we claim that

$$
\left\|u^{\alpha} a(u) f(u)\right\|_{\widetilde{F}(0, t)} \gtrsim t^{\alpha} a(t) f(t), \quad t>0 .
$$

To establish this first let $\alpha>0$, then

$$
\left\|u^{\alpha} a(u) f(u)\right\|_{\widetilde{F}(0, t)} \geq\left\|u^{\alpha} a(u)\right\|_{\widetilde{F}(0, t)} f(t) \sim t^{\alpha} a(t) f(t) .
$$

If, to the contrary, $\alpha \leq 0$

$$
\begin{aligned}
\left\|u^{\alpha} a(u) f(u)\right\|_{\widetilde{F}(0, t)} & =\left\|u^{\alpha+\beta} u^{-\beta} a(u) f(u)\right\|_{\widetilde{F}(0, t)} \geq t^{\alpha+\beta} f(t)\left\|u^{-\beta} a(u)\right\|_{\widetilde{F}(0, t)} \\
& \simeq t^{\alpha+\beta} f(t) t^{-\beta} a(t)=t^{\alpha} a(t) f(t) .
\end{aligned}
$$

This proves our claim. Now, using (9)

$$
t^{\beta} b(t)\left\|u^{\alpha} a(u) f(u)\right\|_{\widetilde{F}(0, t)} \gtrsim t^{\alpha+\beta} a(t) b(t) f(t), \quad t>0,
$$


and therefore

$$
\left\|t^{\beta} b(t)\right\| u^{\alpha} a(u) f(u)\left\|_{\widetilde{F}(0, t)}\right\|_{\widetilde{E}} \geq\left\|t^{\alpha+\beta} a(t) b(t) f(t)\right\|_{\widetilde{E}} .
$$

In order to check (8) choose $F=L_{1}, \alpha=1$ and $\beta=-1$. This concludes the proof.

Remark 3.8. Ultrasymmetric spaces is a large class of r.i. spaces characterized by Pustylnik in [19] as those spaces whose (quasi)-norm is equivalent to

$$
\|f\|_{L_{\varphi, E}}=\left\|\varphi(t) f^{*}(t)\right\|_{\widetilde{E}} .
$$

Here $\varphi$ is a concave function and $\widetilde{E}$ is an r.i. space with respect to the homogeneous measure $d t / t$ on $(0, \infty)$. If the dilation indices of $\varphi$ satisfy that $0<\pi_{\varphi}=\rho_{\varphi}=p<\infty$, then $\frac{\varphi(t)}{t^{p}}, t>0$, is a slowly varying function and $L_{\varphi, E}$ can be seen as the LorentzKaramata type space $L_{p, E, \frac{\varphi(t)}{t^{p}}}$. In these conditions Theorems 3.6 and 3.7 establish equivalent norms for the ultrasymmetric space $L_{\varphi, E}$.

\section{Boundedness of maximal fractional operators and Riesz potential type operators}

In this section we generalize some results of [13] to the framework of LorentzKaramata type spaces. We show applications of Theorem 3.6 to the study of the behaviour of these classical operators. The measure space $(\Omega, \mu)$ will be $\mathbf{R}^{n}$ with the Lebesgue measure. If $a$ is any slowly varying function and $1<p<\infty$, we denote by $\mathcal{M}_{\frac{n}{p}, a}$ the fractional maximal operator

$$
\left(\mathcal{M}_{\frac{n}{p}, a} f\right)(x)=\sup _{x \in \mathcal{Q}} \frac{1}{|\mathcal{Q}|^{1 / p^{\prime}} a(|\mathcal{Q}|)} \int_{\mathcal{Q}}|f(y)| d y,
$$

where the supremum is taken over all cubes $\mathcal{Q}$ in $\mathbf{R}^{n}$ with sides parallel to the coordinates axes. We may let $p=\infty$ if $a$ is equivalent to a measurable decreasing function on $(0, \infty)$.

The decreasing rearrangement of $\mathcal{M}_{\frac{n}{p}, a} f$, for $f \in \mathcal{M}\left(\mathbf{R}^{n}\right)$, is pointwise estimated by the inequality

$$
\left(\mathcal{M}_{\frac{n}{p}, a} f\right)^{*}(t) \lesssim\left\|u^{1 / p} \frac{1}{a(u)} f^{* *}(u)\right\|_{\widetilde{L}_{\infty}(t, \infty)}, \quad t>0,
$$

where the constant only depends on $n, p$ and $a$. See [13] for more information. Now we are in a position to establish the following theorem.

Theorem 4.1. Let $n \in \mathbf{N}, 1<p<\infty, 1<r<p$ and $\frac{1}{q}=\frac{1}{r}-\frac{1}{p}$. Then for any r.i. space $E$ and slowly varying functions $a$ and $b$,

$$
\mathcal{M}_{\frac{n}{p}, a}: L_{r, E, \frac{b}{a}} \longrightarrow L_{q, E, b} .
$$

Moreover, $L_{r, E, \frac{b}{a}}$ is the largest r.i. space mapped by $\mathcal{M}_{\frac{n}{p}, a}$ into $L_{q, E, b}$. then

Proof. First we prove that $\mathcal{M}_{\frac{n}{p}, a}$ is a bounded operator. Let $f \in L_{r, E, \frac{b}{a}}\left(\mathbf{R}^{n}\right)$, 


$$
\begin{aligned}
\left\|\mathcal{M}_{\frac{n}{p}, a} f\right\|_{L_{q, E, b}\left(\mathbf{R}^{n}\right)} & =\left\|t^{1 / q} b(t)\left(\mathcal{M}_{\frac{n}{p}, a} f\right)^{*}(t)\right\|_{\widetilde{E}(0, \infty)} \\
& \lesssim\left\|t^{1 / q} b(t)\right\| u^{1 / p} \frac{1}{a(u)} f^{* *}(u)\left\|_{\widetilde{L}_{\infty}(t, \infty)}\right\|_{\widetilde{E}(0, \infty)} \\
& \sim\left\|u^{1 / r} \frac{b(u)}{a(u)} f^{* *}(u)\right\|_{\widetilde{E}(0, \infty)} \sim\left\|u^{1 / r} \frac{b(u)}{a(u)} f^{*}(u)\right\|_{\widetilde{E}(0, \infty)} .
\end{aligned}
$$

Here we have used Equation (10) and Theorem 3.6. The last equivalence follows from Lemma 2.16 of [9].

In order to show that $L_{r, E, \frac{b}{a}}$ is the largest r.i. space mapped by $\mathcal{M}_{\frac{n}{p}, a}$ into $L_{q, E, b}$, let $f \in \mathcal{M}\left(\mathbf{R}^{n}\right)$ be such that $\mathcal{M}_{\frac{n}{p}, a} f \in L_{q, E, b}$. By Theorem 4.1 of [13], see also [12], there exists a measurable function $h \in \mathcal{M}\left(\mathbf{R}^{n}\right)$ such that

$$
h^{*}=f^{*}, \quad \text { and } \quad\left(\mathcal{M}_{\frac{n}{p}, a} h\right)^{*} \gtrsim\left\|u^{1 / p} \frac{1}{a(u)} h^{* *}(u)\right\|_{\widetilde{L}_{\infty}(t, \infty)} .
$$

Then

$$
\begin{aligned}
\|f\|_{L_{r, E}, \frac{b}{a}} & =\|h\|_{L_{r, E}, \frac{b}{a}}=\left\|t^{1 / r} \frac{b(t)}{a(t)} h^{*}(t)\right\|_{\widetilde{E}} \sim\left\|t^{1 / r} \frac{b(t)}{a(t)} h^{* *}(t)\right\|_{\widetilde{E}} \\
& \sim\left\|t^{1 / q} b(t)\right\| u^{1 / p} \frac{1}{a(u)} h^{* *}(u)\left\|_{\widetilde{L}_{\infty}(t, \infty)}\right\|_{\widetilde{E}} \lesssim\left\|t^{1 / q} b(t)\left(\mathcal{M}_{\frac{n}{p}, a} h\right)^{*}\right\|_{\widetilde{E}}<\infty .
\end{aligned}
$$

This completes the proof.

Similar results can be established for operators of Riesz potential type. Given $1<p<n$ and a slowly varying function $a$ let

$$
\left(I_{\frac{n}{p}, a} f\right)(x)=\int_{\mathbf{R}^{n}} \frac{f(y)}{|x-y|^{n / p^{\prime}} a(|x-y|)} d y .
$$

It is shown in [13], Theorem 4.3, that for all $f \in \mathcal{M}\left(\mathbf{R}^{n}\right)$ and $t>0$

$$
\left(I_{\frac{n}{p}, a} f\right)^{*}(t) \lesssim\left\|u^{1 / p} \frac{1}{a\left(u^{1 / n}\right)} f^{* *}(u)\right\|_{\widetilde{L}_{1}(t, \infty)} .
$$

The inequality constant only depends on $n, p$ and $a$. Now we can establish the following result.

Theorem 4.2. Let $1<p<\infty, 1<r<p$ and put $\frac{1}{q}=\frac{1}{r}-\frac{1}{p}$. Let $a$ and $b$ be slowly varying functions, and let $E$ be an r.i. space. Then for the slowly varying function $\frac{b(t)}{a\left(t^{1 / n}\right)}, t>0$,

$$
I_{\frac{n}{p}, a}: L_{r, E, \frac{b(t)}{a\left(t^{1 / n}\right)}}\left(\mathbf{R}^{n}\right) \longrightarrow L_{q, E, b}\left(\mathbf{R}^{n}\right) .
$$

Moreover, $L_{r, E, \frac{b(t)}{a\left(t^{1 / n}\right)}}\left(\mathbf{R}^{n}\right)$ is the largest r.i. space mapped by $I_{\frac{n}{p}, a}$ into $L_{q, E, b}\left(\mathbf{R}^{n}\right)$.

Proof. Let $f \in L_{r, E, \frac{b(t)}{a\left(t^{1 / n}\right)}}\left(\mathbf{R}^{n}\right)$, then

$$
\begin{aligned}
\left\|I_{\frac{n}{p}, a} f\right\|_{L_{q, E, b}} & =\left\|t^{1 / q} b(t)\left(I_{\frac{n}{p}, a} f\right)^{*}(t)\right\|_{\widetilde{E}} \lesssim\left\|t^{1 / q} b(t)\right\| u^{1 / p} \frac{1}{a\left(u^{1 / n}\right)} f^{* *}(u)\left\|_{\widetilde{L}_{1}(t, \infty)}\right\|_{\widetilde{E}} \\
& \sim\left\|t^{1 / r} \frac{b(t)}{a\left(t^{1 / n}\right)} f^{* *}(t)\right\|_{\widetilde{E}} \sim\left\|t^{1 / r} \frac{b(t)}{a\left(t^{1 / n}\right)} f^{*}(t)\right\|_{\widetilde{E}} .
\end{aligned}
$$

We have used Theorem 3.6 and Equation (11) to establish the above inequality and the following equivalence. See [9] for the last equivalence. 
Similar arguments to those in Theorem 4.1, and Theorem 4.3 of [13], show that $L_{r, E, \frac{b(t)}{a\left(t^{1 / n}\right)}}\left(\mathbf{R}^{n}\right)$ is the largest r.i. space mapped by $I_{\frac{n}{p}, a}$ into $L_{q, E, b}\left(\mathbf{R}^{n}\right)$.

\section{References}

[1] Bennett, C., and R. Sharpley: Interpolation of operators. - Pure and Applied Mathematics 129, Academic Press, Inc., Boston, MA, 1988.

[2] Bingham, N. H., C. M. Goldie, and J. L. Teugels: Regular variation. - Cambridge Univ. Press, Cambridge, 1987.

[3] Brudny̆, Yu. A., and N. Ya. KRUgluak: Interpolation functors and interpolation spaces. Vol. I. - North-Holland Mathematical Library 47, North-Holland Publishing Co., Amsterdam, 1991.

[4] Carro, M. J., S. Ericsson, and L.-E. Persson: Real interpolation for divisible cones. Proc. Edinb. Math. Soc. (2) 42:2, 1999, 285-304.

[5] Cerdà, J., and H. Coll: Function cones and interpolation. - Math. Nachr. 278:3, 2005, $227-239$.

[6] Cerdà, J., and J. Martín: Interpolation of operators on decreasing functions. - Math. Scand. $78: 2,1996,233-245$.

[7] Cerdà, J., and J. Martín: Interpolation of some cones of function spaces. - In: Interaction between functional analysis, harmonic analysis, and probability (Columbia, MO, 1994), Lecture Notes in Pure and Appl. Math. 175, 1996, 145-151.

[8] Cerdà, J., and J. Martín: Interpolation restricted to decreasing functions and Lorentz spaces. - Proc. Edinb. Math. Soc. (2) 42:2, 1999, 243-256.

[9] Cwikel, M., and E. Pustylnik: Weak type interpolation near "endpoint" spaces. - J. Funct. Anal. 171, 2000, 235-277.

[10] Edmunds, D. E., and W. D. Evans: Hardy operators, function spaces and embeddings. Springer-Verlag, Berlin-Heidelberg, 2004.

[11] Edmunds, D. E., and B. Opic: Equivalent quasinorms on Lorentz spaces. - Proc. Amer. Math. Soc. 131, 2003, 745-754.

[12] Edmunds, D. E., and B. OpIC: Boundedness of fractional maximal operators between classical and weak-type Lorentz spaces. - Dissertationes Math. (Rozprawy Mat.) 410, 2002, 1-50.

[13] Edmunds, D. E., and B. OpIC: Alternative characterizations of Lorentz-Karamata spaces. Czechoslovak Math. J. 58:133, 2008, 517-540.

[14] Edmunds, D.E., L. Pick, and R. Kerman: Optimal Sobolev imbeddings involving rearrangement-invariant quasinorms. - J. Funct. Anal. 170:2, 2000, 307-355.

[15] Fernández-Martínez, P., and T. M. Signes: Real interpolation with slowly varying functions and symmetric spaces. - Quart. J. Math. 63:1, 2012, 133-164.

[16] Neves, J. S.: Lorentz-Karamata spaces, Bessel and Riesz potentials and embeddings. Dissertationes Math. (Rozprawy Mat.) 405, 2002, 1-46.

[17] OpIc, B.: New characterizations of Lorentz spaces. - Proc. Roy. Soc. Edinburgh Sect. A 133, 2003, 439-448.

[18] OpIC, B.: On equivalent quasi-norms on Lorentz spaces. - In: Function Spaces, Differential Operators and Nonlinear Analysis. The Hans Triebel Aniversary Volume (edited by D. Haroske, T. Runst and H. J. Schmeisser), Birkhaüser Verlag, Basel/Switzerland, 2003, 415-426.

[19] Pustylnik, E.: Ultrasymmetric spaces. - J. London Math. Soc. 68:2, 2003, 165-182.

[20] SAGHER, Y.: An application of interpolation theory to Fourier series. - Studia Math. 41, 1972, 169-181.

Received 3 December 2012 • Accepted 5 August 2013 